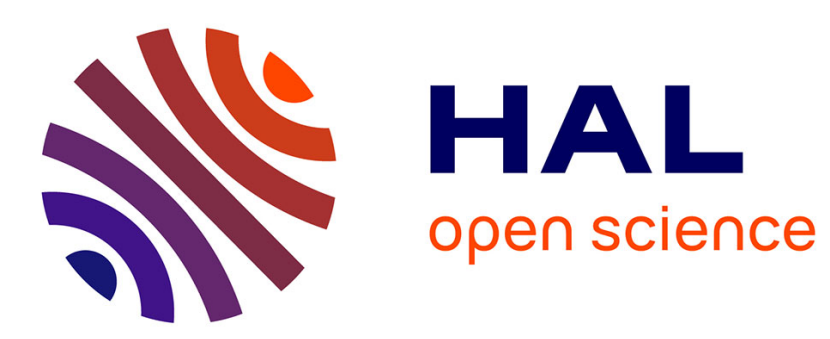

\title{
Habiter la Ville. Interpellation pour l'Eglise en ces temps urbains
}

\author{
Christian Delarbre
}

\section{To cite this version:}

Christian Delarbre. Habiter la Ville. Interpellation pour l'Eglise en ces temps urbains. Transversalités, 2015. hal-02525030

\author{
HAL Id: hal-02525030 \\ https://hal.science/hal-02525030
}

Submitted on 30 Mar 2020

HAL is a multi-disciplinary open access archive for the deposit and dissemination of scientific research documents, whether they are published or not. The documents may come from teaching and research institutions in France or abroad, or from public or private research centers.
L'archive ouverte pluridisciplinaire $\mathbf{H A L}$, est destinée au dépôt et à la diffusion de documents scientifiques de niveau recherche, publiés ou non, émanant des établissements d'enseignement et de recherche français ou étrangers, des laboratoires publics ou privés. 


\section{Habiter la ville \\ Interpellation pour l'Eglise en ces temps urbains. \\ Christian Delarbre - Faculté de théologie de Toulouse.}

\section{Introduction.}

Le phénomène urbain ${ }^{1}$ suppose de la part de l'Eglise une observation particulièrement attentive et une interrogation approfondie sur les questions à portée théologique que soulève cette désormais majoritaire part de l'humanité qui «habite en ville» ${ }^{2}$. Pour la première fois dans l'histoire de l'humanité, la manière d'être au monde de l'humain est urbaine, y compris dans les espace ruraux ${ }^{3}$, au point que Pierre Donadieu puisse employer l'expression de «ruralité urbaine» ${ }^{4}$. L'aspect massif du phénomène urbain et ses implications profondes sur la culture contemporaine, la manière dont les personnes se comprennent, dont leurs relations sont médiatisées par les modes d'être et d'habiter la ville suffisent à nous convaincre de l'importance d'une réflexion théologique et ecclésiologique.

Le phénomène urbain pose d'abord de nombreuses questions anthropologiques qui interrogent immédiatement l'Eglise: celle-ci connaît-elle bien l'homme de la ville, son rapport au temps, à l'espace, aux symboles, à la relation, à l'économie, à la famille, à la différence ? Or, c'est à cet homme-là que l'Eglise est envoyée annoncer l'Evangile au cœur des aspects concrets de son existence urbaine. En réalité, l'Eglise peut aborder avec confiance ces nouveaux rivages humains, pour la bonne raison que sa propre histoire témoigne d'une première diffusion urbaine, non seulement dans les villes mais, et cela appartient aussi à la ville, en cheminant de proche en proche, le long des voies reliant les villes ${ }^{5}$. Bref, l'anthropologie de l'homme urbain n'est pas moins disponible à recevoir l'Evangile que celle de l'homme rural. Mais l'enjeu est d'importance. Soixante ans après, l'avertissement de Henri Mendras est toujours pertinent : «Il ne faudrait pas que l'Eglise apparaisse liée à une civilisation qui meurt [ie. civilisation rurale], ou tout au moins qui n'est plus le

\footnotetext{
1 Parmi l'immense littérature sur la question urbaine, citons: Michel LussAult, L'avènement du monde. Essai sur l'habitation humaine de la Terre, Paris, Éditions du Seuil, 2013, 304p. Olivier MongIN, La condition urbaine. La ville à l'heure de la mondialisation, Paris, Seuil, 2005, 331p. Olivier MongIn, La ville des flux. L'envers et l'endroit de la mondialisation urbaine, Paris, Librairie Anthème Fayard, 2013, 527p. Thierry PAQUOT, Homo Urbanus. Essai sur l'urbanisation du monde et des mours, Paris, Éditions du Félin, 1990, 178 p. Thierry PAQUOT, Michel LUSSAULT, Sophie Body-GENDROT (dir.), La ville et l'urbain l'état des savoirs, Paris, Éditions de la Découverte \& Syros, 2000, 442p. Jean-Pierre PAulet, Géographie urbaine, Paris, Armand Colin/HER, 2000, 315 p. Jean-Marc STÉBÉ, Hervé MARCHAL (dirs.), Traité sur la Ville, Paris, Presses Universitaires de France, 2009, 785 p.
}

${ }^{2}$ Cf. J Véron, «La moitié de la population mondiale vit en ville» Population et Société, n535, juin 2007, publication de l'Institut National d'Etudes Démographiques (disponible sur le site http://www.ined.fr). En 2006, la population urbaine a dépassé les $50 \%$ de la population mondiale, avec de fortes disparités. En France, l'INSEE estime que les pôles urbains et les espaces périurbains couvrent la moitié du territoire et rassemblent $80 \%$ de la population et des emplois (données 2008). Insee Première, $\mathrm{n}^{\circ} 1375$, octobre 2011, p.1.

3 «Dans les pays développés, la nature des polarités changent. Celles des villages diminuent, au profit des bourgs et surtout des villes, dont l'emprise sur les territoires ruraux va en grandissant, grâce à leur accessibilité sans cesse renforcée par la diffusion de moyens de transports modernes. Cette influence accrue de la ville est un élément essentiel de la vie du monde rural dans nos pays», Pierre GILlardot, Géographie rurale, Paris, Ellipses, 1997, p. 188.

4 «La culture rurale est devenue de moins en moins agraire en Europe, sauf dans les pays du Sud. Elle s'est environnementalisée, confrontée à des problèmes de nuisances et de pollutions réelles. Elle s'est patrimonialisée pour répondre à la demande d'identités territoriales des sociétés urbaines. Ces nouvelles valeurs rurales ne peuvent être opposées à la culture urbaine, puisqu'elles sont une conséquence de sa demande de condition d'habitat sédentaire et temporaire à la campagne: une ruralité non agraire mais urbaine, désirée comme un cadre de vie et de loisirs propre aux sports de plein air, à la villégiature et au tourisme.» Pierre DONADIEU, «Campagnes et natures urbaines» p. 157, Thierry PAQUOT, Michel LuSSAUlT, (dir.), op cit.

5 A. DI BerardinO, «L'origine urbana des christianesimo (I-IV secolo)?», Congrés Internacional de Pastoral de les Grans Ciutats (Barcelona, 20-22 maig 2014) [à cette heure, ce Colloque n'est pas encore publié].

Christian Delarbre 
fondement de la civilisation globale. Elle doit dominer le conflit, le sublimer et non y être engagée. ${ }^{6}{ }^{6}$ 'est donc un point fondamental pour l'Eglise de connaître cet homme urbain auquel elle souhaite annoncer l'Evangile.

Parmi toutes ces questions anthropologiques, nous proposons ici de nous intéresser à celle de l'habitat. La ville est en effet un mode particulier d'habiter le monde, en donnant la prééminence aux lieux et aux réseaux sur les territoires et les frontières. La ville est une manière singulière d'agencer les lieux de l'homme en réseaux, et cela sur diverses échelles du local au global. Pour l'Eglise, habiter la ville, c'est alors s'inscrire elle-même comme un réseau de lieux, et inscrire ses lieux propres dans le réseau de la ville. Plus que de ses territoires et de ses limites paroissiales, l'Eglise doit s'interroger sur les lieux qu'elle habite, qu'elle anime, qu'elle bâtit dans la ville et sur leur manière d'être agencés en réseaux et reliés au réseau de la ville, ou réseau qu'est la ville.

\section{1) Des lieux et des réseaux.}

Le mode d'habiter est une des ruptures anthropologiques principales que connaît le monde urbain. Naturellement, l'habitat est un thème central de l'urbanisme et un vaste champ de recherche et d'expérimentation des urbanistes. Les modes d'habitat dans la ville, d'ailleurs variés (du bidonville à la gentrification des centres villes) jouent tous une profonde transformation de l'être au monde. Habiter la ville est une autre manière d'habiter le monde. En particulier, tout habitat de l'homme (et même de toute espèce animale) est une certaine façon de déterminer certains lieux ${ }^{7}$ et la manière de les parcourir et de les partager. Le monde urbain peut ainsi être compris comme une certaine manière d'agencer les lieux en réseaux.

Considérons donc la ville comme un ensemble de lieux déterminés, dont un bon nombre sont des inventions de la ville: la place, la rue, plus récemment le square, le parc, le musée, l'hypermarché... Une des caractéristiques de cet ensemble varié est la séparation fonctionnelle des lieux: l'urbain habite, travaille, s'amuse, court, nage, skie, retrouve ses amis, commerce... en des lieux généralement différents, et parfois fort lointains les uns des autres.

Ces lieux sont indissociables du réseau qui les unit. Le réseau urbain est une des caractéristiques les plus frappantes de la ville, avec la multiplication des lieux qu'il relie. Le réseau est matériel (voies de circulation, eau, électricité, fibres ou antennes internet...) et immatériel (entrelacs des contacts humains, influences de la cité, liens symboliques, procédés d'identification). Les souffrances dans la ville s'inscrivent d'ailleurs dans les lieux qui, pour diverses raisons, sont insuffisamment reliés aux autres, ou qui n'ont pas achevé leur processus d'intégration dans le réseau urbain de manière matérielle ou immatérielle. La relégation d'un quartier peut provenir d'un déficit de connexion matérielle mais surtout d'un manque d'identification à la ville et à sa collectivité, et la «nature contrainte de l'entre soi» ${ }^{8}$.

\footnotetext{
6 Henri MENDRAS, «L'influence des divers courants migratoires sur la vie religieuse des campagnes» p. 159, dans Fernand Boulard et alii, Paroisses urbaines, paroisses rurales, Paris, Casterman, 1958, 222 p.

7 Christian Delarbre, «L’Eglise en lieux et en réseaux», Documents Episcopat, n4/2008. Dans cet article, je définis un lieu comme une «portion déterminée de l'espace», l'élément de détermination étant une activité humaine. Ce qui fait un lieu d'Eglise est ainsi une détermination théologique: un lieu, où, à son échelle, se réalise ce qui fait l'Eglise. Au moins quelques fidèles, réunis par la proclamation de la parole et la louange de Dieu en ce lieu, et reliés à la communion apostolique. Autre caractéristique importante, les lieux sont «discrets» au sens mathématique du terme, c'est-à-dire qu'ils sont discontinus.

8 Jacques DonZELOT, «La ville à trois vitesses: relégation, périurbanisation, gentrification», p.19 dans Esprit, $\mathrm{n}^{\circ} 303$, mars-avril 2004.
}

Christian Delarbre 
Lieux et réseaux sont hiérarchisés, du plus local (mon appartement, mon épicerie du coin, mon lieu de travail ou mon école, la place où je retrouve mes amis) au plus global (la gare, l'aéroport, le stade, le "haut lieu" d'identification qu'est un monument) L'Eglise elle-même est dans la ville comme un ensemble de lieux reliés (ou non...) les uns aux autres. C'est pourquoi le territoire a moins de sens en ville et dans la vaste agglomération que l'agencement des lieux et leur manière d'être en réseau et en connexion avec le reste de la ville. Il convient donc de s'interroger sur les lieux d'Eglise dans la ville. Ils sont à différentes échelles: du local au global, d'une salle de rencontre à une cathédrale, d'un domicile à un lieu de culte ou à une université catholique. Et ils sont reliés par des réseaux matériels et immatériels.

Mais il faut avoir conscience que si la ville est un ensemble de lieux en réseaux, l'homme urbain, lui, parcourt la cité selon des itinéraires qui lui sont propres. Il vit ces lieux selon ce qui lui convient, il joue de ces lieux, parfois même en les détournant ${ }^{9}$. Gardons-nous, en dessinant des «cartes pastorales», d'oublier que les personnes vivent des itinéraires qui leur sont propres. La pluralité des lieux et leur mise en réseau est une manière de privilégier l'itinéraire à la carte ${ }^{10}$.

Le mode d'habiter propre à la ville interpelle donc l'Eglise et sa propre manière «d'habiter». Le rapport théologique entre l'Eglise et le fait d'habiter est profond. Il trouve ses sources dans la promesse d'une terre faite au peuple. Dans le renversement par lequel le Seigneur se bâtit lui-même une maison, maison de David, plutôt que de laisser le roi lui construire un temple. Dans la révélation finale par laquelle «il a habité parmi nous et nous avons vu sa gloire» ${ }^{11}$. Dans la manifestation de l'Eglise Une, en une réalité «locale» ou «particulière» qui accomplit sa mission d'annonce et de célébration en des lieux concrets.

La manière dont l'Eglise habite la ville et ce monde urbain nous interroge tout d'abord sur les lieux que l'Eglise pourra habiter. Nous avons écrit ailleurs qu'il n'y a pas de «lieux favorables» prédéterminés à devenir des lieux d'Eglise, mais à l'inverse qu'est lieu d'Eglise l'endroit où ce qui fait l'Eglise est présent et se manifeste: lieu du rassemblement des fidèles (même deux ou trois), lieux de l'annonce de la Parole, lieu de la célébration, autour du ministère apostolique. Lieux qui participent à des échelles diverses et de manière plus ou moins complète à la réalisation de l'Eglise ici et maintenant ${ }^{12}$. L'Eglise a bien des lieux en ville: non seulement les édifices du culte, mais encore: évêchés, salles paroissiales, presbytères, couvents, lieux d'accueil, écoles, studios de télé ou de radio, antennes du Secours Catholique et ainsi de suite. La vie de l'Eglise dans la ville passe par cette pluralité de lieux car c'est ainsi qu'est le territoire urbain: un réseau complexe de lieux divers parcourus de façon en principe libre et autonome par la population ${ }^{13}$.

Comment ces lieux sont-ils (ou ne sont-ils pas suffisamment) en lien avec le reste de la ville, en lien physique, mais aussi en lien immatériel (symbolique, numérique, communicationnel)? De vastes portions du réseau urbain ne sont-ils pas étrangers à ces lieux de manifestation du salut que

\footnotetext{
9 «S'il est vrai qu'un ordre spatial organise un ensemble de possibilités (par exemple, par une place où l'on peut circuler) et d'interdictions (par exemple, par un mur qui empêche d'avancer), le marcheur actualise certaines d'entre elles. Par là, il les fait être autant que paraître. Mais aussi il les déplace et il en invente d'autres puisque les traverses, dérives ou improvisations de la marche, privilégient, muent ou délaissent des éléments spatiauX» Michel DE CERTEAU, L'invention du quotidien. 1. arts de faire, Paris, Gallimard, 1990, p.149.

${ }^{10}$ Michel DE CERTEAU, op.cit,, p.177.

${ }^{11} \mathrm{Jn} 1,14$.

12 Cf. Christian Delarbre, Théologie du Lieu. Pour une Eglise à taille urbaine, t.I, Lille, ANRT, 2006, p.422. Les éléments constitutifs de l'Eglise sont la portion du peuple de Dieu, la Parole proclamée, la célébration de l'Eucharistie, autour du ministère apostolique. Il y a «de l'Eglise» dans les lieux où tout ou partie de ces éléments sont présents.
}

${ }^{13}$ Ibid. p.744.

Christian Delarbre 
sont les lieux d'Eglise ? A quel niveau (domicile, quartier, agglomération, monde) ces lieux existent-ils, entre les hauts lieux tel que Lourdes ou la Place Saint-Pierre et le lieu de la communauté de base réunie à domicile pour prier et partager la Parole ? Et comment des lieux d'Eglise se trouveront sur le parcours du passant? Comment participeront-ils à des itinéraires de croyants ? Et quelle annonce de l'Evangile est opportune en chacun de ces lieux? Certains lieux sont-ils à orienter vers certaines formes particulières d'annonce? Quels lieux d'Eglise sont à inventer en conformité avec l'anthropologie urbaine?

\section{2) Trois formes de l'habitat urbain.}

La maison est le premier lieu de la territorialité des personnes ; elle est « leur coin du monde $»^{14}$. Elle est le premier jalon de la territorialité, et de ce point de vue, elle se confond avec leur propre intériorité. Demeure de l'homme, elle est le lieu où l'homme demeure, et demeure en lui-même. Le lieu d'habitation a ainsi une importance considérable non seulement sur les conditions matérielles d'existence de l'habitant, mais sur sa propre perception de la condition humaine.

De façon plus précise, Jacques Donzelot distingue trois types d'habitats, et finalement, trois façons d'habiter la ville : la relégation, la périurbanisation, la gentrification ${ }^{15}$. Il s'agit fondamentalement d'un processus complexe de séparation qui affecte la ville depuis un quart de siècle ${ }^{16}$. Le phénomène de relégation concerne les grands ensembles urbains dans lesquels " les habitants (...) forment une société particulière marquée par la nature contrainte de l'entre soi qui caractérise leur relation au sens où le libre choix figure pour une part bien minime dans la constitution de leur voisinage $»^{17}$. Toutefois, cela ne signifie pas que les relations soient absentes ou n'existent que sur un mode négatif ${ }^{8}$. Le confinement social de ce type d'habitat engendre un phénomène d'immobilité. «C'est le repli dans le logement, la hâte de se retrouver derrière sa porte fermée qui confère à ces cités un tel caractère d'immobilité, comme si sortir, bouger, aller ailleurs et revenir, était une épreuve que l'on s'imposait le moins possible. $»^{19}$ Ainsi la relégation du logement entraîne un phénomène d'isolement entretenu, qui finit même par être revendiqué comme identité.

Le second type d'habitat est décrit par le phénomène de périurbanisation. Surtout à partir des années soixante-dix, cette phase de la croissance urbaine a adjoint à la ville des espaces ruraux où, par une politique d'accès à la propriété, se sont massivement construites des maisons

14 Cf. p.97 in Georges Di MÉO, «Les fondements humains de la territorialité », p. 95-139, in Géographie sociale et territoires. Pour mieux cerner les enjeux territoriaux contemporains: de la ville et du pays rural au monde, des Étatsnations aux identités ethniques et régionales, Paris, Nathan, (coll. « Géographie/fac. »), 2001, 317p. Di Méo s’inspire ici de Gaston BACHELARD, La poétique de l'espace, Paris, PUF, (coll. « Quadrige » n²4), 1998 (C1957), 214 p.

15 Jacques DONZELOT, « La ville à trois vitesses : relégation, périurbanisation, gentrification », Esprit, 303/3-4, (2004), p. 14-39. Cf aussi. J.-P. PAUlet, "Les centres : crises et rénovations », p. 138-161, " De la banlieue à la périurbanisation », p. 162-180, in Géographie urbaine, Paris, Armand Colin, 2000, 315 p.

${ }^{16}$ Cette complexité des facteurs économiques, culturels, juridiques dans la division de l'habitat est brièvement présentée dans Marcel RonCAYOLO, "Les comportements résidentiels ", p. 124-127, dans La ville et ses territoires, Paris, Gallimard, 1990 (C1978), 285 p.

17 Jacques DONZELOT, art. cit., p. 19.

18 «Des études fines ont montré que, même dans les cités les plus stigmatisées, si une partie des habitants vit sa localisation comme un sujet d'opprobre, une autre, non moins importante, s'approprie cette localisation, en fait une part positive de son identité. », ibid., p. 19. Des remarques similaires chez David LEPOUTRE, Cour de banlieue. Codes, rites et langages, Paris, Éditions Odile Jacob, 2001, C1997, 459 p.

19 Jacques DONZELOT, art. cit., p. 21.

Christian Delarbre 
individuelles ${ }^{20}$. Ce déplacement vers la périphérie est lié à un rejet de la ville dense, de son encombrement et de sa densité exigeant de s'accommoder de la présence des autres. En outre, cet habitat compense la vie mobile par la stabilité, le sentiment de permanence, la présence apaisante d'une nature sans doute mythifiée, bien différente en tout cas de la nature telle que perçue par les voisins ruraux qui en tirent leur subsistance. Le pavillon de banlieue typique des lotissements périurbains est entouré en France d'une grille ou mieux d'un mur, ainsi que d'un jardin ; cela forme autant de coquilles protectrices vis-à-vis de l'extérieur. C'est un lieu protégé et protecteur, dont l'accès est strictement contrôlé, réservé aux membres de la famille, et à ceux qui sont invités explicitement.

Troisième mode pour demeurer dans la ville, la gentrification. Elle concerne les classes aisées qui habitent ou reviennent habiter dans les centres-villes. Le phénomène de périurbanisation, s'il se justifie par la recherche de qualité de vie et de sécurité, est aussi lié à des phénomènes économiques: s'éloigner de la ville est nécessaire à celui qui recherche un logement suffisamment vaste pour sa famille, désormais inabordable en centre-ville. La gentrification est un retour en centre-ville de la «partie supérieure de ces couches moyennes $»^{21}$. Les habitants des centres gentrifiés bénéficient de la proximité de ce qui leur importe là où ils vivent, et de la capacité de se projeter en n'importe quel point du globe, physiquement ou virtuellement, si nécessaire. Il s'agit de jouir des avantages de la ville sans en subir les inconvénients.

3) Eléments d'une pastorale de l'habitat urbain.

Donzelot, par cette tripartition schématique de l'habitat, met en évidence la logique de séparation comme une tendance forte de la ville moderne. Entre ces trois pôles d'habitat, peu de communication sociale s'avère possible. Les habitants vivent dans la même ville, mais ne vivent clairement pas la même ville. Cette réalité sociale engendre d'importantes conséquences pastorales. D'une part, le lieu d'habitation lui-même apparaît comme un niveau essentiel des lieux d'Eglise, d'autre part, la séparation de ces habitats indique à «l'Eglise communion» un aspect essentiel de sa mission: réunir ce qui est séparé, contribuer à renverser les nouveaux «murs de la haine» par la croix du Christ ${ }^{22}$.

A ce niveau premier du territoire urbain qu'est l'habitat, le seul lieu d'Église qui puisse exister est le lieu d'habitation lui-même. Nous pouvons donc nous demander si la maison périurbaine, avec ses valeurs de protection parfois excessives, et d'accès maîtrisé, ses valeurs d'autonomie et de qualité de vie, sa contrainte de mobilité, peut être un lieu d'Église. L'appartement dans une cité, avec son voisinage imposé, avec la coexistence ethnique et culturelle que suppose cet habitat, avec ses contraintes d'immobilité, peut-il être un lieu d'Église ? L'appartement de standing, avec son accès contrôlé, avec la fenêtre virtuelle ou réelle sur le monde de ceux qui peuvent prétendre que « la planète est un gros village », avec son digicode et ses loyers exorbitants peut-il être un lieu d'Église?

\footnotetext{
${ }^{20}$ Marie-Chirstine JAILLET, « Peut-on parler de sécession urbaine à propos des villes européennes ? », Esprit, 258/11, (1999), p. 145-167. ID. « L'espace périurbain, un univers pour les classes moyennes », Esprit, 303/3-4, (2004), p. 40-62.

${ }^{21}$ Ibid., p. 31. Cf. aussi Jean-Pierre PAULET, « Un processus de “gentryfication ” », p. 147-148, in Géographie urbaine, Paris, Armand Colin, 2000, 315 p.

${ }^{22}$ Nous nous intéressons au niveau de l'habitat domestique. Ce travail pourrait être complété par une réflexion sur les questions pastorales concernant le quartier et l'agglomération. Cf. Christian DELARBRE, op. cit. pp.757-789.
} 
Ils le seront d'abord d'une même façon. Le lieu d'Église a quelque chose à voir en effet avec un sanctuaire de la présence de Dieu, avec l'événement de l'action de salut du Christ ${ }^{23}$. C'est-à-dire que le lieu n'est pas lieu d'Église du fait de ce qu'il est géographiquement, socialement, financièrement, mais parce qu'il est un lieu où le Seigneur vient faire sa demeure. De ce point de vue, la triple caractérisation de ces différents habitats ne dit rien: la manifestation du salut ne dépendent en rien du lieu où vient le salut en Christ. Paradoxalement, cette indifférence est l'aspect essentiel du lieu d'Église ici. Où que l'on vive, Dieu peut venir y faire sa demeure, et ce faisant, transformer radicalement la demeure du fidèle au milieu de ses semblables : le voilà jeté comme un « étranger » par l'étrangeté de l'Évangile qui vient habiter en sa demeure. Pour le chrétien, sa façon d'habiter peut donc être grandement changée par une telle découverte : elle devient le lieu d'une conversion, dans les rapports de voisinage par exemple.

Ainsi, le domicile est un lieu d'Église d'abord comme le lieu où l'on demeure, lieu d'intériorité et de prière ${ }^{24}$. La chambre fermée de la prière est la métaphore physique de la Présence divine accessible au croyant où qu'il soit, pourvu qu'il adore en esprit et en vérité, métaphore du Seigneur qui vient établir en 1 'homme sa demeure ${ }^{25}$. Elle est le lieu privilégié de la présence en notre existence de celui qui l'a transcendé.

Elle est encore un lieu d'Église parce qu'elle est le lieu de la cellule familiale chrétienne qui relève du mystère d'alliance. Mgr Barbarin, alors qu'on l'interroge sur le nombre nécessaire de lieux de culte à La Duchère, importante cité lyonnaise, répond par la nécessité de renforcer «l'homme intérieur » en donnant l'exemple de la vie familiale, où le foyer devient lieu de culte ${ }^{26}$.

La demeure est aussi pour le chrétien de la ville un lieu d'Église où il porte témoignage : en effet, elle peut être un lieu ouvert, un lieu d'accueil tranchant avec la tendance à la paranoïa et à l'isolement que dénote l'architecture de banlieue. Sans aller jusqu'à ouvrir leur porte aux quatre vents, les chrétiens de la ville ne rechignent pas à accueillir chez eux groupes de prières ou de partage de vie. La question de l'insertion de leur demeure dans le voisinage devient une réelle question pastorale et spirituelle. De ce point de vue encore, la maison du chrétien est véritablement un lieu d'Église, à la fois comme lieu de la Parole et témoignage de la foi.

Par ailleurs, c'est un lieu privilégié de la pastorale des sacrements : en effet, le ministre ou l'agent pastoral dit beaucoup sur les sacrements ou rites demandés par une famille (baptême, mariage, funérailles, communion et sacrement aux malades) s'il se déplace et pénètre dans la maison. Pénétrer à de tels moments dans la demeure familiale ouvre une possibilité de quitter l'aspect trop exclusivement fonctionnel que peuvent avoir souvent les demandes, pour les replacer comme autant d'étapes sur un chemin de foi et d'occasions de rencontre avec Celui qui se rend Présent au milieu de nous. L'acteur pastoral considère le foyer qu'il visite comme un lieu d'Église non d'abord du fait de sa présence, mais en affirmant dans la foi que le Seigneur l'a précédé en ce lieu, qu'il vient en rendre témoignage au nom de l'Église par sa visite, et à en manifester le lien avec toute l'Eglise.

\footnotetext{
${ }^{23}$ La manifestation du salut en un lieu est l'expérience de la présence du Christ par l'Esprit qui est médiatisée par la prédication de la Parole, la vie sacramentelle et liturgique, la transformation des relations humaines, la naissance d'une portio du Peuple de Dieu, et par la mise en relation de façon ministérielle de chaque lieu particulier avec la communion ecclésiale.

24 « Pour toi, quand tu veux prier, entre dans ta chambre la plus retirée, verrouille ta porte et adresse ta prière à ton Père qui est là dans le secret. », Mt 6, 6 .

${ }_{25}$ Nous renvoyons à l'étude de Gaston BACHELARD, La poétique de l'espace, Paris, Presses Universitaires de France, (coll. «Quadrige »), 1998 (C1957), 214 p. Le philosophe établit la maison comme métaphore de l'intériorité.

${ }^{26}$ p. 69 dans : Laurent LARCHER, Cités de l'espérance. En banlieue, parmi les nouveaux aventuriers de Dieu, Paris, Cerf, 2006, 172 p.
}

Christian Delarbre 
On ne négligera pas non plus de promouvoir les célébrations domestiques, spécialement les bénédictions : elles proclament la présence salvifique de Dieu au plus proche de nous, elles invitent à ouvrir ce qui peut n'être qu'une coquille protectrice finalement aliénante ; elles portent aussi une parole de réconfort et de guérison face aux angoisses que le lieu où l'on demeure médiatise souvent ; elles portent la présence du Seigneur en des lieux qui semblent parfois abandonnés des hommes.

A ces aspects pastoraux communs aux différentes formes d'habitats, il faut ensuite reconnaître ce qui peut être propre à une pastorale des quartiers contraints, périurbain ou gentrifiés. Brièvement, une pastorale de l'habitat contraint ne peut éviter le temps long du compagnonnage, la proximité de la communauté d'appartenance, la solidarité concrète de la communauté de base. L'Eglise y contribue au patient tissage de liens de voisinage, de liens interculturels et d'engagements collectifs devant les contraintes communes à ces habitats. Pour l'habitat périurbain, la pastorale sera davantage marquée par la consommation de services, mais aussi par la variété des propositions centrées sur le cheminement individuel. Le fort investissement affectif des habitants du périurbain dans leur maison (qui s'est traduit souvent par des considérables sacrifices financiers) se traduit pastoralement par l'importance des réunions de groupes au domicile des différents membres. Enfin, aisément ouvertes sur le reste du monde, les demeures de l'habitat gentrifié, pour être des lieux d'Église, gagneront à s'ouvrir davantage aux réalités proches des autres habitants de la ville, à participer à l'animation chrétienne des centres, lieux d'intense passage.

Pour élargir ces réflexions de théologie pratique sur l'habitat urbain au-delà de l'horizon occidental, citons brièvement le travail et l'expérience de Benjamin Bravo, de Mexico, qui développe la notion d'Eglise domestique (Iglesia de casa ${ }^{27}$. B. Bravo note que cette Iglesia de casa peut prendre plusieurs formes et appeler plusieurs pastorales. Il propose ainsi l'Eglise domestique communauté ecclésiale de base, à la fois manière exemplaire de vivre l'Evangile dans le quotidien, et précieuse pour permettre aux plus pauvres ou plus modestes d'être acteurs de l'Eglise. Puis l'Eglise domestique affinitaire, regroupant des personnes partageant une même culture, une même situation sociale, ou une même génération. Ou l'Eglise domestique définie par une demande (parents d'enfants catéchisés, famille d'un malade). Et enfin l'Eglise domestique «religion populaire», qui se réunit à l'occasion d'un événement, d'un rite, d'une coutume. Une réflexion pastorale approfondie à partir de l'habitat des urbains engendre ainsi une attention particulière à des modes de vie ecclésiaux les plus proche de la «demeure des hommes», Eglise domestique, ou foyer Eglise.

Ces brèves réflexions permettent d'ouvrir sous divers angles la pastorale à la réalité complexe de l'habitat urbain avec ses diverses manières de «demeurer dans le monde». Il existe cependant un niveau inférieur encore dans l'échelle les lieux de la ville, le lieu des sans-lieux si l'on peut dire. Là, l'Eglise est aux marges de la ville, où le rêve urbain se transforme en cauchemars, auprès des laissés pour compte et des exclus de la ville, auprès des sans-lieux qui n'en sont pas moins habités, à l'instar de tous les autres citoyens, de ce même rêve qui les retient dans la cité.

4) Des flux et des écrans.

Dans cette dernière partie, nous voudrions considérer la part plus immatérielle de l'habitat urbain. En plus des lieux physiques ou il demeure, lieux reliés de façon matérielle par des voies de

\footnotetext{
${ }^{27}$ Benjamin BRAVO, «El tejido eclesial y los tejidos urbanos (sociales, economicos, culturales, religiosos)» mais aussi Luca BRESSAN, «Individuo, persona, comunità nella grande città» dans Congrés Internacional de Pastoral de les Grans Ciutats (Barcelona, 20-22 maig 2014) [non publié à cette date].
} 
communication, l'homme urbain est encore connecté à un vaste réseau immatériel dont l'importance ne fait que croître. L'ouvrage d'Olivier Mongin, La ville des flux ${ }^{28}$ aborde justement les mutations que les réseaux, spécialement immatériels, portent à la manière d'être au monde, au mode d'habiter, à la relation au lieu que l'être humain entretient avec son espace. L'auteur emploie le terme de flux (plutôt que réseau) pour désigner les connexions matérielles mais surtout immatérielles qui conditionnent les échanges multiples dont la ville est faite. Plutôt que le binôme «lieu et réseau», Mongin parle des écrans qui nous permettent d'accéder à ces flux et d'y participer. Selon lui, la conséquence principale de cette «société des écrans» est assez pessimiste. Exacerbant le désir d'ubiquité et d'instantanéité, le risque de ce phénomène est «d'accorder un moindre degré d'attention au monde ${ }^{29}$. En outre, le rapport aux écrans modifie la relation entre «un dedans et un dehors». Mélangeant les espaces privés et publics, les flux et leurs écrans modifient en profondeur la manière d'habiter la ville, mais aussi d'habiter le monde. Mongin évoque d'ailleurs trois sortes d'écrans: écrans de cinéma, écrans de télévision et écrans d'ordinateur. Notons que chacun renvoie bien en définitive à un certain type de lieu, hiérarchisé ou fonctionnellement séparé, au sens de ce que nous indiquions plus haut.

Retenons qu'aussi immatériel soit le réseau, aussi complexes et indélimitables soient les flux, il n'empêche que l'être humain, personne corporelle, ne peut pas ne pas habiter le monde en un lieu, ne serait-ce que le lieu de son corps. La véritable mutation est sans doute que des personnes, séparées en leur lieu, puissent être réunies par les flux. Prenons l'exemple de réunions de travail. De plus en plus, les participants se retrouvent par l'intermédiaire de ces flux et de ces écrans. Physiquement séparés, chacun en son lieu, et pourtant réunis au point de mener ensemble un projet. Même des chirurgiens parviennent à opérer à distance pourtant dans le lieu même du corps du patient. Nous savons l'importance de la messe télévisées pour de nombreuses personnes âgées ou malades, chez elles ou en maison de retraite. Des réseaux comme Skype permettent à des familles de se réunir à distance. Nous remarquons encore la capacité des jeunes à être là non seulement avec d'autres personnes présentes physiquement, mais en même temps avec des amis connectés, menant plusieurs conversations simultanément.

Cette interconnection est à la fois fascinante et effrayante: fascinante par sa complexité technique et sa facilité d'usage, effrayante car nul n'en mesure encore les conséquences anthropologiques. Mongin est plutôt pessimiste: «La crise de l'horizon géographique et historique, la dissolution des frontières entre privé et public participent d'un marché de la relation et d'un desserrement de l'expérience spatio-temporelle, celle que rend possible la circulation dans le réseau virtuel. Un tel desserrement se traduit par une avidité de maîtrise des informations, par la recherche de la vitesse, par la fuite en avant et la perte du sens de la latéralité» ${ }^{30}$. Une virtualisation qui menacerait radicalement la manière même d'être au monde.

Il n'est pas certain qu'il faille être si alarmiste. La complexification des flux et la mise en réseau d'une part croissante de l'humanité sont aussi bien la poursuite d'une universalisation de celle-ci, et de sa conscience globale. En outre, aussi connecté soit-on au réseau, nous ne sommes pas dématérialisés pour autant. Notre conscience au monde reste notre corps jeté dans l'espace et le temps. Il nous faut bien un «écran», nouvelle expression de la paradoxale «nuée lumineuse», qui cache et révèle à la fois, c'est-à-dire finalement bien un lieu où cette virtualité se projette pour nous rejoindre et interagir avec nous. De fait, son travail sur les flux conduit Mongin à s'interroger à son tour sur le lieu. Il propose trois expressions : non-lieux, hyperlieux et milieux pour aborder les nouvelles relations à l'espace induites par les flux.

\footnotetext{
${ }^{28}$ Olivier Mongin, La ville des flux, op. cit. p.89.

${ }^{29}$ Ibid. p.89.

${ }^{30}$ Ibid. p.93.
}

Christian Delarbre 
Le non-lieu est une réalité virtuelle et déterritorialisée décrivant les agissements marginaux des pirates de la toile ou d'autres réseaux obscurs trouvant intérêt au «brouillage des limites» ${ }^{31}$. L'auteur note: «la mise en réseau produit, du fait de son opacité, des zones imperceptibles de nondroit, d'insécurité et d'incertitudes propices à la disjonction et aux blocages.» ${ }^{32}$ Ces non-lieux relèvent d'une forme de blocage ou d'appropriation des échanges. Le blocage des échanges virtuel vise pourtant bien le blocage des échanges tout court: économiques, sociaux, communications, transports. Au plan pastoral, nous pouvons ici reconnaître les tentatives et tentations d'Eglises «virtuelles», d'Eglises «interstitielles» et cellulaires qui profitent de l'opacité des réseaux virtuels. Notons l'usage immodéré de ces ressources immatérielles par des groupuscules souvent peu signifiants par eux-mêmes, mais qui se donnent une visibilité et une importance très loin de la réalité «corporelle». Leur objet est bien de brouiller les frontières, leur stratégie est bien de bloquer les flux. Leur dérive sectaire est certaine. Leur danger réel. Il me parait significatif que ces formes de détournement des réseaux refusent absolument le lieu.

Les hyperlieux consistent à «se manifester à la face du monde, se montrer, s'exhiber et, de l'autre, il faut s'interconnecter et donc se déterritorialiser par rapport à l'espace de proximité $)^{33}$. L'auteur prend l'exemple des villes de tours et de gratte-ciels, d'îles ou de citadelles. L'Eglise ne construit pas de tours comme à Dubaï, mais elle est friande de manifestations massives ou d'espaces symboliques. Une part de la pastorale contemporaine peut s'appuyer ainsi sur les nouvelles technologies de communication pour organiser et promouvoir des événements très visibles (JMJ), ou pour favoriser la fréquentation d'espaces symboliques (hauts lieux de pèlerinages ou patrimoniaux, Lourdes, le Mont Saint-Michel) mais, comme dit Mongin, ces hyperlieux sont «déterritorialisés par rapport à l'espace de proximité». Leurs conditions de réalisation sont une vaste interconnection qui n'hésite pas à se manifester, véritables «vitrines globalisée» (encore une expression de Mongin). Enfin, l'auteur signale une fragilité de ces hyperlieux qui ne peut qu'interpeler l'Eglise: ces réalités tendent à dissocier «une manifestation exacerbée du global et un repli identitaire sur le local».

Troisième réalité mixte de réel et de virtuel, les milieux sont «des émergences singulières qui rendent possible le passage du virtuel au réel en favorisant le surgissement de lieux qui sont des places vides» ${ }^{34}$. Les échanges virtuels se prolongent en «réalisation» dans un ici et maintenant occupant une «place» laissée vide jusqu'alors. Des réseaux sociaux débouchent quelque part, «trouvent une place où rendre effectifs et corporels les échanges de tous ordres». L'auteur prend l'exemple des printemps arabes, mais de façon plus quotidienne et moins conflictuelle, notons que les jeunes usent beaucoup de cette manière de «mise en lieu»: les échanges de la cour de récréation ou de la soirée passée ensemble se prolongent d'autre manière sous forme d'échanges virtuels, qui eux-mêmes donneront littéralement «lieu» à de nouveaux moments de rencontre, à d'autres événements. On se retrouve alors sur une place, dans une rue, et souvent un de ces interstices, un de ces espaces laissés en déshérence par les autres habitants de la ville.

Ces trois réalités sont autant de manières d'inventer un nouveau rapport du dedans et du dehors, du privé et du public, du virtuel et du réel, de réinventer la dimension spatiale de l'existence. Mongin nous alerte: si les flux s'avèrent plus forts que les lieux, ils les reconfigurent selon les deux premières tendances décrites plus haut. Les lieux de l'Eglise, que nous devons continuer de concevoir dans leur réalité matérielle, avec un ici et maintenant, et que nous avons dit être reliés en

\footnotetext{
${ }^{31}$ Ibid. p.137.

${ }^{32}$ Ibid. p.138.

${ }^{33}$ Ibid. p. 135 .

${ }^{34}$ Ibid. p. 134.
}

Christian Delarbre 
réseaux immatériels de relations, d'identifications, de symboliques, se trouvent, comme les autres lieux de la ville, reconfigurés par ces nouveaux flux de nouvelles possibilités, pas toutes souhaitables. Mais il est illusoire de vouloir tenir à l'écart de ces flux les lieux d'Eglise traditionnels. Retenons en particulier cette notion de milieux: au service d'échanges initiés de manière immatérielle, les lieux d'Eglise dans la ville demeurent des lieux de territorialisation et de réalisation concrète des relations.

\section{Conclusion.}

$\mathrm{Au}$ terme de ce parcours, nous reconnaissons combien la réflexion anthropologique sur le mode d'habiter la ville enrichit la compréhension de l'homme en général, mais aussi des fidèles du Christ, massivement urbains, qui constitue le Peuple de Dieu. Cet effort de compréhension ici juste balbutiant apparait indispensable pour que l'Eglise réponde à sa mission d'évangélisation en notre temps. L'urbain porte décidément une profonde mutation de la manière d'être au monde, à l'autre, et donc à Dieu. Pourtant, ces changements me paraissent moins porteurs d'angoisses que de promesses. Cette humanité urbaine est bien autant intéressante, fascinante, aimable au bout du compte, que celle des temps ruraux et des sociétés stables. La Révélation s'achève par une humanité rassemblée en une ville unique, immense, et aux multiples portes. Et cette humanité à laquelle la Jérusalem céleste est promise n'est pas autre que la multitude qui parcourt et habite nos villes. Comme le Pape François nous y invite, osons poser un regard de contemplation sur cette réalité urbaine, sur la ville et ses habitants ${ }^{35}$. 